Working Paper 11-24

Economic Series

July, 2011
Departamento de Economía Universidad Carlos III de Madrid

Calle Madrid, 126 28903 Getafe (Spain)

Fax (34) 916249875

\title{
Temptation, Horizontal Differentiation and Monopoly Pricing
}

\author{
Joaquín Gómez Miñambres \\ July 25,2011
}

\begin{abstract}
We study the implications for pricing strategies and product offerings of consumers' temptation when the differentiation of the product is horizontal. With horizontal differentiation, the temptation state is represented by a change in the consumers' ideal product on the Hotelling line, so that consumers have two (possibly distinct) ideal products: one when committed and another when tempted. The firm faces the following trade-off: for the consumer who diverge the most between the ideal product with temptation and commitment, if the firm positions a product close to the consumer's temptation ideal product, it increases the consumer's surplus when tempted but decreases surplus with commitment, which lowers the consumer's incentive to participate. This paper shows that, because of this trade-off, the firm may exclude products that are too close to the temptation preferences in the optimal menu. Moreover, it is shown that product diversity and firm's profits decrease with the probability of temptation and with the consumers' awareness of their dynamic inconsistency. (JEL: D11, D42, D82, L11, L12, L15)
\end{abstract}

Keywords: Temptation; Commitment; Price discrimination

\footnotetext{
"Department of Economics, Universidad Carlos III de Madrid, C./ Madrid, 126, 28903 Getafe (Madrid), Spain. E-mail: jgminamb@eco.uc3m.es. I am exceedingly grateful to my supervisors $\mathrm{M}^{\mathrm{a}}$ Ángeles de Frutos and Susanna Esteban for their support and encouragement. In addition, the paper has benefitted from the comments of Lapo Filistrucchi, Patricio Dalton as well as participants at the ENTER Jamboree Conference, 2010 and the audiences at several seminars. All remaining errors are attributable to me.
} 


\section{Introduction}

Consumers' temptation is an important characteristic of the consumer purchasing behavior. Many consumers establish ex-ante that they would like to commit to consuming healthy, low calorie groceries. Nonetheless, ex-post temptation takes place and they modify their choices towards unhealthier alternatives. This behavior reflects the dynamic inconsistency of consumers' preferences commonly known as temptation. An important implication is that when consumers are aware of their future change in preferences, they are more willing to enter stores which do not carry unhealthy products in order to avoid ex-post choices inconsistent with ex-ante preferences.

To capture this idea, Strotz (1955) and Kreps (1979) introduced a class of preferences known as the "temptation representation". Let $M$ denote the consumers' choice set and $U$ the utility function - the commitment utility function - that he has when making the shopping list. The consumer anticipates that, once inside the store, his utility function will change to $V$ with a positive probability $\pi$. Let $x^{u}$ denote the consumer's choice with $U$-his commitment choice- and $x^{v}$ denote his choice with $V$ -his tempting choice. Then, the consumer's expected ex-ante utility is given by

$$
W(M)=(1-\pi) U\left(x^{u}\right)+\pi U\left(x^{v}\right) .
$$

In this setting a dynamic consistency problem arises because, while the consumer would like to commit ex-ante to choosing $x^{u}$, with probability $\pi$, he ends up choosing $x^{v}$. We can interpret this preference representation as though the consumer had, ex-post, two different possible selves: a tempted and a committed self. ${ }^{2}$

Although simplified, this representation is convenient for our purposes as it creates an ex-ante demand for menus that implement commitment. ${ }^{3}$ Eliaz and Spiegler (2006) considers a similar consumer representation.

\footnotetext{
${ }^{2}$ Note that this representation is quite extreme: if an agent falls into temptation he will forget about the commitment preferences and choose according to the temptation preferences only.

${ }^{3}$ Some papers have studied other temptation representation. Gul and Pessendorfer (2001), for instance, consider that consumer's utility from a choice set equals the realized commitment utility
} 
A novel contribution of this paper is that we analyze a monopolist's optimal pricing problem when the product is horizontally differentiated. This allows us to capture temptation as a change in the consumer's ideal product on the Hotelling line. We consider a continuum of consumer types; each consumer type knows that he has two (possibly distinct) ideal products on a Hotelling line, one when committed and another one when tempted. In the basic model, we assume that all consumer types have the same temptation ideal product, located at one extreme of the Hotelling line, but they differ when committed. Continuing from our previous example, this assumption means that while consumers prefer products with different calories when committed, they are only tempted by high calories product. Later in the paper, as an extension, we study a generalization of the temptation representation.

In our model, a monopolist sells several products that can differ in their location on the Hotelling line and in pricing. Therefore, the firm's problem is to decide which goods to offer on the Hotelling line and charge a price for each one in such a way that expected profits are maximized. Since consumers are aware of the dynamic inconsistency of their preferences, when designing the optimal selling strategy the firm has to worry about both their incentives to enter the store (ex-ante $I R$ ), and their incentives to participate once inside (ex-post IR). Moreover, the firm must ensure that once inside the store, each consumer chooses the product designed for himself minus the linear "self-control costs", i.e., the realized temptation utility minus the maximum value of the temptation utility over the choice set. Fudenberg and Levine (2005) allow for non-linear selfcontrol costs, as they argue that, with this model we can consider self-control as a limited resource such that the "cognitive load" leads to agents falling into temptation more easily. Dekel, Lipman and Rustichini's (2005) representation covers situations in which agents face uncertainty about the "strength of temptation". Finally, under the Chatterjee and Krishna (2006) representation, the agent has two different selves and temptation is thought of as the choice of a "virtual alternate self" or alter ego, who appears with a positive probability which depends on the choice set. If the temptation probability is not menu-dependent this representation coincides with the one that we use in our model. 
$($ ex-post $I C){ }^{4}$

Using this model, we can understand the relationship between the consumers' temptation and the firm's optimal product design. Does temptation increase or decrease product diversity? Do prices increase with temptation? Does it have welfare implications?

In the standard horizontal differentiation model without temptation, the monopolist offers the ideal product of each consumer type. In our model, instead, the firm faces the following trade-off: by positioning products closer to the temptation preferences, it can increase its profits under the temptation state. However, it also decreases the consumers' ex-ante utility, especially for those with a greater distance between their ideal products with commitment and temptation. Therefore, the firm's optimal menu may exclude products that are too close to the temptation preferences since otherwise these consumers will not derive sufficient utility from entering the store. In equilibrium, two types of consumers coexist: consumers with similar preferences in the two states, who always consume the same product, and consumers with most diverging preferences, who consume different products in different states. The size of the two consumer groups, which is given by the degree of product diversity, and the firm's profits decrease with the probability of temptation.

In recent years, several authors have explored the implications of consumer temptation on pricing. A paper related to our work is Esteban, Miyagawa and Shum (2006). Using Gul and Pessendorfer's (2001) preferences and a vertical differentiation environment, they construct a model in which a monopolist chooses the price and quality of the goods it offers the consumer once inside one store. Thus, as in the present paper, they restrict the number of menus to one. As in our model, they find that the firm is not better off when the consumer operates under temptation

\footnotetext{
${ }^{4}$ Eliaz and Spiegler (2004) use a model in which consumers and the firm have to sign a contract before entering the store, thus they work with an ex-ante incentive compatibility constraint. In our model we do not consider the possibility of the ex-ante contract, so consumers must decide, ex-post, which product to choose from among all the available offers in the store's menu.
} 
and that the heterogeneity of the product offered may be bounded as a result. In other related papers, such as Esteban and Miyagawa (2006a), the firm can offer multiple menus which allow the firm to earn more profits, while Esteban and Miyagawa (2006b) consider a competitive framework in which each firm can also choose several menus. Also using a vertical differentiation setting, Eliaz and Spiegler (2006) study a model in which dynamically inconsistent agents sign a contract with a firm, using the same temptation representation used here. In contrast with our model, they assume that while the firm correctly anticipates the consumers' inconsistency, consumers incorrectly believe that, with some probability, they are going to take actions in accordance with their "commitment" preferences. This "non-common priors" assumption is the source of the exploitative contracts that arise in equilibrium. In contrast, our model assumes that consumers are perfectly aware of their dynamic inconsistency, i.e., they are "sophisticated" consumers. However, in Section 5, we extend our model to consider "naive" consumers, as in the Eliaz and Spiegler model. We show that when consumers are sufficiently naive, the monopolist can extract all the consumers' surplus. The monopolist offers a menu which would not be accepted by consumers if they had the same priors as the firm. They enter the store attracted by the benefits of an "imaginary offer" that they (incorrectly) believe to be purchasing when committed.

There are other papers that study mechanism design for consumers with time inconsistent preferences, however they focus on present-biased preferences with hyperbolic discounting. For instance, O’Donoghue and Rabin (1999b) study the optimal contract that the firm must offer to a worker who is naive, i.e., he is unaware of his dynamic inconsistency. DellaVigna and Malmendier (2004) study a model with two kinds of goods: investment goods with immediate costs and delayed benefits, and leisure goods with immediate benefits but delayed costs. In equilibrium, firms price investment goods below marginal costs and leisure goods above marginal costs. If consumers are fully aware of their dynamic inconsistency, the agents achieve a so- 
cially efficient solution, but if agents are naive then the equilibrium is inefficient. Sarafidis (2005) constructs a model in which consumers form expectations not only about their future behavior but also about the firm's prices. The main result is that the more naive consumers are, the lower the monopolist profits will be.

Finally, in contrast to our model some papers considers that the temptation probability is an endogenous variable. For instance, there are papers that study addition and consumers with environmental cues. Laibson (2001), use a version of the Becker and Murphy (1988) model of addition, including environmental cues that arise during each period with some probability. Due to a cue, the more the agents consume today, the greater the marginal utility tomorrow. Therefore, consumption is determined by the stock of past consumption in each state of the world. Their results show that the probability of falling into consumption due to a cue (temptation) decreases with the consumers' discount factor, the value of the outside option, and the impact of current consumption on the stock of consumption. Our model is not about environmental cues since in the consumers' representation used here, the temptation probability is an exogenous variable.

The paper proceeds as follows. Section 2 describes the basic model. In Section 3, we study two important benchmarks: the time consistent preferences case, i.e., the standard model in which the temptation probability is zero, and the time inconsistent preferences case in which, ex-post, the only possible state is the temptation state. Section 4 characterizes the monopolist's optimal menu. Section 5 extends the model to cover the case of non-common priors and more general specifications of the consumers' preferences. Finally, Section 6 concludes.

\section{The Model}

A monopolist can produce and sell several products in one store. Products are horizontally differentiated à la Hotelling on a segment of unit length. An offer from the 
seller is denoted by $x$ and is a pair $(q, p)$, where $q \in[0,1]$ represents the product's location and $p \in \mathbb{R}_{+}$its price. A menu $M$ is a compact subset of offers $M \subseteq \mathbb{R}^{2}$. Let $\bar{M}=M \cup(\phi, 0)$ denote the set of offers available to the consumer, where $(\phi, 0)$ is the outside option which has a price of zero. We assume consumers are perfectly informed about the offers in the menu.

There is a set of consumers, each of them buying at most one product and deciding, ex-ante, whether to enter the monopolist's store. They can also choose to stay outside the store, which we formalize as choosing the outside option $N E=(\phi, 0)$.

To model consumers' behavior we use the "dual-selves" approach based on Strotz (1995) and Kreps (1979). In particular, we consider that, before entering the store, a consumer evaluates his ex-post decisions with his commitment utility function $U(\cdot)$. However, with a probability $\pi$, a consumer makes ex-post decisions with his temptation utility function $V(\cdot)$.

We assume that $\theta$ is the consumer's commitment ideal product on the Hotelling line which is distributed according to a uniform distribution with support $[0,1]$. We also assume that $\theta^{v}=0$ is the consumers' ideal product when tempted. ${ }^{5}$ Therefore, we consider that, when tempted, all consumers have "extreme" preferences. ${ }^{6}$ Then, given a typical menu $M$, the (ex-ante) surplus of a consumer type $\theta$ if he enters the store is:

$$
W(\bar{M} ; \theta)=(1-\pi) \max _{x \in \bar{M}} U(x, \theta)+\pi U\left(x^{v}, \theta\right),
$$

where

$$
x^{v}=\underset{x \in \bar{M}}{\arg \max } V(x) .
$$

A consumer type $\theta$ enters the store only if his ex-ante utility is positive. We call this condition ex-ante IR. The interpretation is that, once a consumer is inside the store, he will choose with probability $(1-\pi)$ the best element in the menu with his commitment utility, while with probability $\pi$ he will choose with his temptation

\footnotetext{
${ }^{5}$ In Section 5 we study more general specifications of the temptation preferences.

${ }^{6}$ Note that $\theta^{v}=1$ has a similar interpretation.
} 
utility. From now on we will refer to the former as the commitment state and to the latter as the temptation state.

Given a menu $\bar{M}$, we define the assignment function $x(\cdot)=(q(\cdot), p(\cdot)):[0,1] \rightarrow$ $\bar{M}$ that specifies for each type $\theta \in[0,1]$ the offer that he is expected to choose.

Definition 1 An offer $x(\theta)=(q(\theta), p(\theta))$ is the commitment choice for consumer $\theta$ if $x(\theta)=\underset{x \in \bar{M}}{\arg \max } U(x ; \theta)$.

Definition 2 An offer $x\left(\theta^{v}\right)=x^{v}=\left(q^{v}, p^{v}\right)$ is the tempting choice for the consumer if $x^{v}=\underset{x \in \bar{M}}{\arg \max } V(x)$.

Therefore, the tempting (resp., commitment) choice is the offer that a consumer is expected to choose in the temptation (resp., commitment) state. Note that since consumers have the same ideal product when tempted, their choices under this state must be the same. On the other hand, under commitment, consumers have different preferences so their choices may differ.

We consider the following specification for the commitment and temptation utility functions, respectively:

$$
\begin{aligned}
U(x ; \theta) & =s-p-t(q, \theta), \\
V(x) & =s-p-t\left(q, \theta^{v}\right),
\end{aligned}
$$

and normalize

$$
U((\phi, 0) ; \theta)=V((\phi, 0))=W(N E ; \theta)=0
$$

This is the typical utility specification of a horizontal differentiation model, where $s \in \mathbb{R}_{+}$represents the maximum possible surplus enjoyed by consumers, and $t(q, \theta)$ represents the transportation cost, which satisfies:

(i) Symmetry: $t(q, \theta)=t(\theta, q)$;

(ii) Non negativity: $t(q, \theta) \geq 0$;

(iii) Identity of indiscernibles: $t(q, \theta)=0$ iff $q=\theta$; 
(iv) Increasing in Euclidean distance: $t\left(q_{1}, \theta_{1}\right) \geq t\left(q_{2}, \theta_{2}\right)$ iff $\left|\theta_{1}-q_{1}\right| \geq\left|\theta_{2}-q_{2}\right|$; and,

(v) Strict Superadditivity: $t(q, \theta)>t(q, z)+t(z, \theta)$ for all $z \in(q, \theta)$.

Property $(i)-(i v)$ are standard properties used in any horizontal differentiation model. An important implication of $(v)$, which we use in our analysis, is that $\left|t_{1}(q, \theta)\right|>\left|t_{1}(q, z)\right|$ for all $z \in(q, \theta)$. Hence, the marginal transportation cost increases in the distance between $\theta$ and $q \cdot{ }^{7}$ An example of a transportation cost function which satisfies all properties $(i)-(v)$ is the quadratic function: $t(q, \theta)=(q-\theta)^{2}$.

For simplicity, we assume the market is fully covered, which, as standard, requires $s$ being sufficiently large.

\section{The monopolist's problem}

The monopolist's problem is to design a menu $\bar{M}=\left\{x(\theta), x^{v}\right\}_{\theta \in[0,1]}$ which maximizes his profits subject to consumers' participation and incentive compatibility constraints. For simplicity, we assume that the marginal cost of production is equal to zero. Therefore, the monopolist's problem is given by

$$
\max _{\left\{x(\theta), x^{v}\right\}_{\theta \in[0,1]}}(1-\pi) \int p(\theta) d \theta+\pi p^{v}
$$

s.t., for all $\theta \in[0,1]$,

$$
\begin{gathered}
W(\bar{M} ; \theta)=(1-\pi) U(x(\theta) ; \theta)+\pi U\left(x^{v} ; \theta\right) \geq 0, \\
U(x(\theta) ; \theta) \geq 0, \\
V\left(x^{v}\right) \geq 0, \\
U(x(\theta) ; \theta) \geq U(y ; \theta) \text { for all } y \in M, \\
V\left(x^{v}\right) \geq V(y) \text { for all } y \in M .
\end{gathered}
$$

(Ex-post $V$-IC)

\footnotetext{
${ }^{7}$ Note that this works as a single crossing condition for all consumer types with $\theta>q$.
} 
Consumer type $\theta$ will enter the store if $W(\bar{M} ; \theta) \geq 0$. So, when the ex-ante $I R$ constraint is satisfied, consumers choose from $\bar{M}{ }^{8}$ The ex-post $I R$ and $I C$ are standard constraints. As usual, ex-post $I R$ says that a consumer is at least as well off purchasing from the menu as choosing the outside option. Finally, ex-post $I C$ says that a consumer of type $\theta$ cannot be better off by pretending to be another type in each state of the world.

The ex-post $I R$ and $I C$ constraints together imply that $x(\theta)$ (resp., $x^{v}$ ) is an optimal choice for $U(\cdot, \theta)$ (resp., $V(\cdot))$.

In the following lemma we show that if the ex-ante $I R$ and ex-post $U-I C$ constraints are satisfied, consumers obtain a non-negative ex-post surplus.

Lemma 1 Ex-ante IR and ex-post $U-I C$ imply ex-post $U-I R$.

Proof. Ex-post $U-I C$ tells us

$$
U(x(\theta) ; \theta) \geq U(y ; \theta) \text { for all } y \in M .
$$

Since the market is covered, $x(\theta) \in M$ and thus equation (4) implies that for all $\pi \in[0,1]$

$$
U(x(\theta) ; \theta) \geq(1-\pi) U(x(\theta) ; \theta)+\pi U\left(x^{v} ; \theta\right)=W(M ; \theta) \geq 0,
$$

where the second inequality follows from ex-ante $I R$.

By Lemma 1 we know that, since the market is covered, when the monopolist designs the optimal menu he only needs to worry about ex-ante $I R$, ex-post $U-I C$ and ex-post $V-I R$.

\footnotetext{
${ }^{8}$ Thus, it is assumed that if a consumer $\theta$ is indifferent to choosing from $\bar{M}$ or $N E$, i.e., if $W(\bar{M} ; \theta)=0$, he ends up choosing from $\bar{M}$.
} 


\section{Benchmark Cases}

In this section we study two benchmark specifications that are particular cases of our model. First, we study the case in which the temptation probability equals zero. That is, consumers' choices are always consistent. Second, we study the case in which the temptation probability equals one. That is, all consumers anticipate that they will always be tempted inside the store.

\subsection{Time Consistent Preferences $(\pi=0)$}

Our utility representation allows for consumers to be evaluating both their ex-ante and their ex-post decisions with their commitment utility. This is the case when the temptation probability equals zero, so that our model becomes a standard horizontal differentiation model. In this standard model, by selling the set of consumers' ideal products, the firm can extract the entire surplus of each consumer type. Thus, in equilibrium, the monopolist will offer the menu $M^{F C}=\{\theta, s\}$ for all $\theta \in[0,1]$. This is obviously a feasible menu: All $I R$ constraints are binding while all the $I C$ constraints are slack.

However, as the temptation probability increases, the monopolist faces the following trade-offs. Since the market is covered, product $q^{v}$, which is to be consumed when consumers are tempted, must be desirable ex-post, satisfying ex-post $V-I R$, but must be such that consumers also want to enter the store with their ex-ante utility function. If product $q^{v}$ is located closer to $\theta^{v}$, then it is the furthest away from the commitment ideal products of some of the remaining consumers, and thus prices must be lower for the ex-ante $I R$ of these consumer types to be satisfied. This effect gives the monopolist fewer incentives to position $q^{v}$ at $\theta^{v}$. But then, consumer types closer to $\theta^{v}$ will consume the tempting product under commitment as well, which implies 
that the monopolist narrows the variety of products he offers when the temptation probability $(\pi)$ increases.

\subsection{Time Inconsistent Preferences $(\pi=1)$}

Assume now that the probability of temptation is such that consumers, once inside the store, will purchase the tempting choice. Therefore, the monopolist only sells $q^{v}$ and his maximization problem becomes

$$
\max _{\left\{q^{v}, p^{v}\right\}} p^{v}
$$

s.t., for all $\theta \in[0,1]$,

$$
\begin{array}{rlrl}
U\left(x^{v} ; \theta\right) & \geq 0, & (\text { Ex-ante } I R) \\
V\left(x^{v}\right) \geq 0 . & (\text { Ex-post } V-I R)
\end{array}
$$

Since the market is covered, the ex-ante $I R$ constraint must be satisfied for all

$\theta \in[0,1]$. As $\frac{d U\left(x^{v} ; \theta\right)}{d \theta} \leq 0(\geq 0)$ for all $\theta \geq q^{v}\left(\theta \leq q^{v}\right)$, the binding constraints are the ex-ante $I R$ constraint for $\theta=1$ and the ex-post $V-I R$ constraint. It is then immediate to see that the monopolist maximizes profits by positioning $q^{v}$ equidistantly between $\theta^{v}=0$ and $\theta=1$. Thus, $q^{v}=\frac{1}{2}$; and $p^{v}$ is set to extract the consumers' entire ex-post surplus. In equilibrium, the monopolist offers the menu $M^{F T}=\left\{\frac{1}{2}, s-t\left(\frac{1}{2}, \theta^{v}\right)\right\}$.

In sum, if $\pi=0$, the monopolist offers the consumers' ideal products, whereas if $\pi=1$, the monopolist offers a single product.

\section{Characterization of the Optimal Menu}

In this section we compute the optimal menu of offers for any $\pi$. We start by deriving some auxiliary results. 
Note that ex-post incentive compatibility and superadditivity of $t(q, \theta)$ imply $q(\widehat{\theta}) \geq q(\theta)$ for all $\widehat{\theta} \geq \theta$. Moreover, since $\theta^{v}=0$, it follows trivially that if $x(\theta)=x^{v}$ for some consumer type $\theta \in[0,1]$, then $x(\widehat{\theta})=x^{v}$ for all $\widehat{\theta} \leq \theta$. Therefore, consumers purchasing the same product in both states (i.e., $x(\theta)=x^{v}$ ) are located closer to the temptation preferences, $\theta^{v}$, than consumers who purchase different products in different states (i.e., $x(\theta) \neq x^{v}$ ). Let us denote by $\theta_{k}$ the lowest consumer type who buy the same product in both states. In the following lemma we prove that the firm optimally offers the ideal commitment product for all consumer types with $\theta \geq \theta_{k}$.

Lemma 2 At the optimal menu $q(\theta)=\theta$ for all $\theta \in\left[\theta_{k}, 1\right]$. Moreover, $p(\theta)=p^{*} \geq$ $p^{v}$, for all $\theta \in\left[\theta_{k}, 1\right]$.

The intuition behind Lemma 2 is that by offering the ideal commitment product for all consumer types $\theta \in\left[\theta_{k}, 1\right]$, their ex-ante surplus is increased. This allows the monopolist to raise the prices of the products it offers.

In equilibrium we have that $x(\theta)=x^{v}$ for all $\theta<\theta_{k}$ while $x(\theta) \neq x^{v}$ for all $\theta \geq \theta_{k}$. For consumer $\theta_{k}, U\left(x\left(\theta_{k}\right) ; \theta_{k}\right)=U\left(x^{v} ; \theta_{k}\right)$.

Moreover, by Lemma 2, we obtain the following monotonicity result:

$$
\text { for all } \theta \geq q^{v}\left(\theta \leq q^{v}\right), \frac{d W}{d \theta} \leq 0\left(\frac{d W}{d \theta} \geq 0\right)
$$

The next lemma shows that the tempting choice coincides with the commitment choice of consumer $\theta_{k}$.

Lemma 3 In equilibrium, $x^{v}=\left(\theta_{k}, p^{*}\right)$.

By Lemma 1 and Lemma 2, it is obvious that, if $V\left(x^{v}\right)>0(\operatorname{resp} ., W(\bar{M} ; 1)>0)$, it is profitable for the monopolist to increase $q^{v}$ (resp., decrease $q^{v}$ ) and increase $p$. Therefore, in equilibrium, $V\left(x^{v}\right)=W(\bar{M} ; 1)=0$ must hold. Then, 


$$
s-p^{*}-t\left(q^{v}, 0\right)=(1-\pi)\left(s-p^{*}\right)+\pi\left(s-p^{*}-t\left(q^{v}, 1\right)\right)=0,
$$

and

$$
t\left(q^{v}, 0\right)=\pi t\left(q^{v}, 1\right)
$$

Based on the auxiliary results above, the next proposition fully characterize the optimal menu.

Proposition 1 At the optimal menu, the products offered are $x^{*}=\left(\theta, p^{*}\right)$, for all $\theta \in\left[\theta_{k}, 1\right]$.

Furthermore, the tempting choice $\left(q^{v}, p^{v}\right)=\left(\theta_{k}, p^{*}\right)$ satisfies

$$
t\left(q^{v}, 0\right)=\pi t\left(q^{v}, 1\right)
$$

and

$$
p^{v}=p^{*}=s-t\left(q^{v}, 0\right) .
$$

Note that these results give us noteworthy features of the optimal menu. Firstly, $q^{v}$ is an increasing function of $\pi$ with $q^{v}(\pi=0)=0$ and $q^{v}(\pi=1)=\frac{1}{2}$, which coincides with the full commitment and the full temptation benchmarks, respectively. Secondly, since by Lemma $2, q^{v}=\theta^{k}$, as temptation probability increases fewer consumers make different choices under different states, so product diversity decreases.

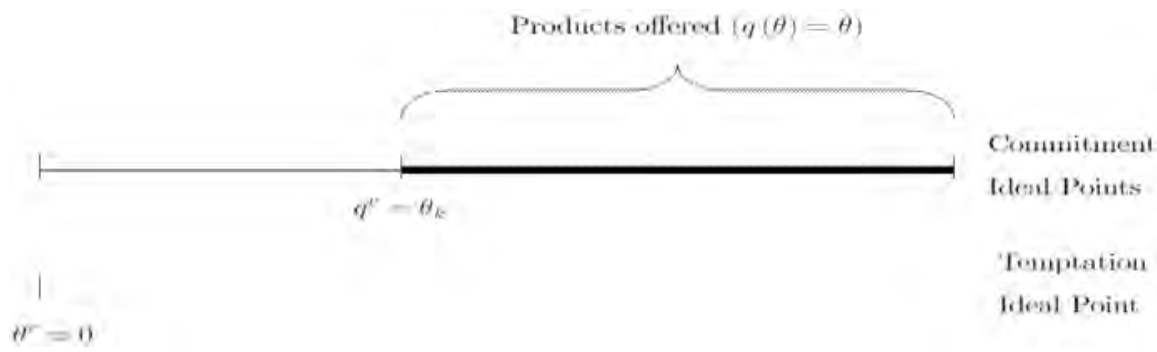

Fig. 1. Optimal Menu 
Moreover, since all products sold in the store are sold at the price $p^{*}$ and the market is covered, the firm's profits are

$$
\Pi=p^{*}=s-t\left(q^{v}, 0\right) .
$$

Thus, product prices and monopolist's profits decrease with $\pi$. The intuition is that since consumers are aware of their time inconsistency, a higher temptation probability has to be compensated with lower prices to attract consumers into the store, which leads to lower profits for the monopolist.

Finally, the consumers' ex-ante surplus is

$$
W(\bar{M} ; \theta)=\left\{\begin{array}{cc}
t\left(q^{v}, 0\right)-t\left(q^{v}, \theta\right) & \forall \theta \in\left[0, \theta_{k}\right] \\
\pi\left[t\left(q^{v}, 1\right)-t\left(q^{v}, \theta\right)\right] & \forall \theta \in\left[\theta_{k}, 1\right]
\end{array}\right.
$$

By Lemma 2, we know that $q^{v}=\theta_{k}$, thus $\theta_{k}$ is the only consumer type who consumes his ideal product under both states, so it is not surprising that he gets the maximum consumer surplus of all the consumer types.

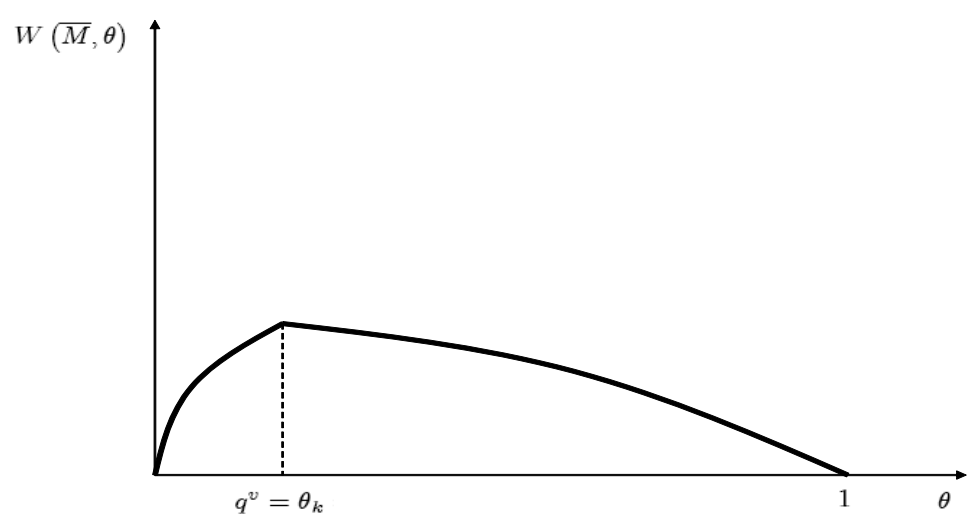

Fig. 2. Ex-ante consumers' surplus

Moreover, we know that a higher temptation probability implies lower product prices to attract consumer $\theta=1$ into the store (i.e., $W(\bar{M} ; 1)=0$ needs to hold) 
which implies that for all $\theta \in(0,1), W(\bar{M} ; \theta)$ increases with the temptation probability.

\section{$5 \quad$ Extensions}

In this section we analyze two important extensions of our previous model. First, we extend our setting to allow for non common priors. Second, we relax the assumption that $\theta^{v}$ is located at one extreme of the Hotelling line.

\subsection{The Non-Common Priors Case}

It is reasonable to think that in several situations, the firm has better knowledge about consumers' change in tastes than consumers themselves. Like Eliaz and Spiegler (2006) (ES from here on), we formalize this non-common priors idea assuming that the firm knows that temptation occurs with probability one, while consumers believe that their preferences will not change with a positive probability $(1-\pi)$ (i.e., they are naive). Therefore, the monopolist now only cares about the profits from the tempting choice $x^{v}=\left(q^{v}, p^{v}\right)$ and uses the commitment choices to induce consumers to enter the store (i.e., as a hook).

Since the monopolist wants to charge the highest $p^{v}$, in this setting we still have $V\left(x^{v}\right)=0$ in equilibrium, thus from the $V-I C$, it follows that

$$
0=V\left(x^{v}\right) \geq V(x)=s-p-t\left(q, \theta^{v}\right)
$$

Moreover, the monopolist wants to charge the lowest possible $p$ to induce consumers to enter the store, which is $p=s-t\left(q, \theta^{v}\right)$.

In the next lemma we show that the monopolist offers just one product to be consumed under commitment. 
Lemma 4 At the optimal menu there is a single commitment choice for consumers given by $x=(1, s-t(1,0))$. Consequently, $\theta_{k}=0$.

The intuition behind Lemma 5 is that the firm wants to locate the commitment offer as far from the tempting choice as possible in order to charge the highest possible price under the temptation state. This implies that in equilibrium the commitment choice is so attractive for committed consumers that all consumers $\theta \in(0,1]$ expect to choose $x$ under commitment.

Since the monopolist wants to charge the highest possible $p^{v}$, we want to check whether charging $p^{v}=s$ (i.e., locating $q^{v}=\theta^{v}=0$ ) is feasible. The next lemma addresses this issue and summarizes the equilibrium tempting choice.

Lemma 5 At the optimal menu,

(i) $\quad x^{v}=\left(q^{v}, p^{v}\right)=(0, s)$ for all $\pi \leq \frac{1}{2}$, and,

(ii) $q^{v}$ is given by the equation, $t\left(q^{v}, 1\right)-t\left(q^{v}, 0\right)=\frac{1-\pi}{\pi} t(1,0)$ for all $\pi>\frac{1}{2}$.

When consumers believe that temptation occurs with a low probability $\left(\pi \leq \frac{1}{2}\right)$, the firm can extract the consumers' entire surplus, positioning $q^{v}=0$. Note that, by the properties of the transportation cost function, the solution $q^{v}(\pi)$ is continuous and $\frac{d q^{v}(\pi)}{d \pi}>0$ for all $\pi>\frac{1}{2}$. Moreover, when $\pi=1$, we obtain the full temptation benchmark with common priors. Figure 3 shows the equilibrium choices under both types of priors. 


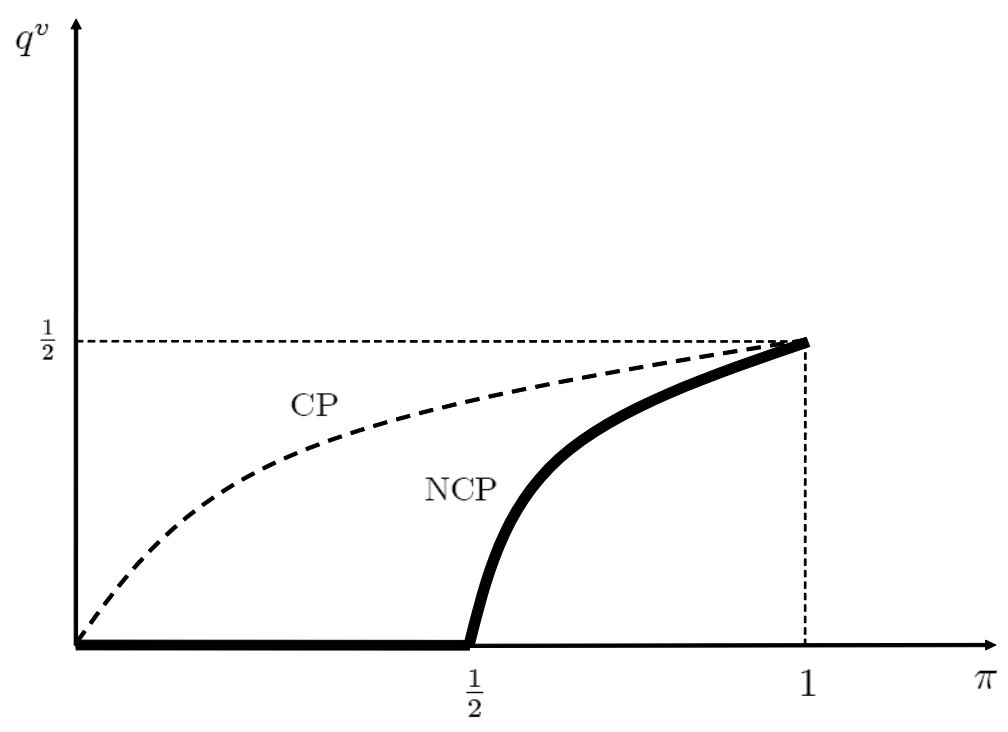

Fig. 3. Equilibrium with Common Priors (CP) and Non-Common Priors (NCP).

Therefore our results are similar in spirit to that of ES. Note that $x$ is an "imaginary offer" (or a hook): consumers believe that they will purchase it with a positive probability, whereas the firm knows that all consumers will end up purchasing $x^{v}$ once in the store. The monopolist uses the imaginary offer to attract consumers into the store and it wants to charge the lowest possible $p$. However, this price has a lower bound $(p=s-t(1,0))$ due to the incentive compatibility constraints. When consumers believe that temptation occurs with a low probability $\left(\pi<\frac{1}{2}\right)$ the monopolist can extract all of the consumers' surplus when they are tempted, and consumers find it optimal to enter the store due to the benefits that they (incorrectly) expect to receive when committed. However, when $\pi$ is sufficiently large, the expected gains that consumer $\theta=1$ expects to obtain under commitment are lower than the expected losses that he expects to suffer when tempted if the tempting choice is $x^{v}=(0, s)$. Because of this, the monopolist has to design $q^{v}$ sufficiently close to $\theta=1$; otherwise, this consumer type will not derive a sufficiently high ex-ante utility to entice him to 
enter into store. ${ }^{9}$

In our non-common priors case, the monopolist is offering a menu which would not be accepted by some consumer types if they had the same priors as him. Therefore, it is obvious that the monopolist obtains higher profits in the non-common priors case than in the common priors case. Moreover as with common priors, the price of the tempting choice, and hence the monopolist's profits, decrease with the temptation probability.

Let us now discuss the differences between our analysis and that of ES. As in the present paper, ES study a model in which a monopolist has to design a menu for dynamically inconsistent consumers. However, whereas here we consider a horizontal differentiation model in which consumers differ in their commitment preferences, ES study a vertical differentiation model in which consumers differ only in their prior beliefs about the future state. In ES equilibrium, sophisticated types (i.e., those with a high prior) choose a contract which perfectly commits them to their commitment choice, while naive types (i.e., those with a low prior) choose an exploitative contract, which is a contract that gives them a negative utility under temptation state in accordance with their commitment preferences (i.e., $\left.U\left(x^{v}\right)<0\right)$. Our results confirm that with a low $\pi$ there is exploitation in equilibrium (i.e., $U\left(x^{v}\right)<0$ for all $\theta>0$ ). However, with our temptation model we can provide more results. If $\pi$ is high, the exploited consumers are those for which the difference between their ideal product when committed, $\theta$, and the temptation ideal product, $\theta^{v}$, is large. Moreover, the set of exploited consumers decreases as $\pi$ increases, because the firm has to design the tempting choice closer to their commitment preferences.

\footnotetext{
${ }^{9}$ As in the common priors case we are assuming that $s$ is sufficiently large to cover the market. However in this case there is an important difference: when $\pi<\frac{1}{2}$, the firm wants to cover the market for any $s$, the reason is that in this case the firm can attract even consumer $\theta=1$ by offering $x^{v}=(0, s)$.
} 


\subsection{Generalization of Temptation Preferences}

In this section, we relax the assumption that consumers' temptation ideal product is a single point located at the extreme of the line. Firstly, we consider the case where the consumers' temptation ideal product is located in the interior of the interval $[0,1]$. Secondly, we study the case where, ex-ante, the agents face uncertainty about the ex-post temptation ideal product.

\section{General temptation ideal product $0 \leq \theta^{v} \leq 1$}

We next show that our previous results with $\theta^{v}$ located at one extreme of the Hotelling line can be easily extended to the case where $0 \leq \theta^{v} \leq 1$. To understand this, we next consider the case where $\theta^{v}=\frac{1}{2}$. Note that we can interpret this case as if faced with two different standard cases where $\theta^{v}$ is located at one extreme, one with $\theta \in$ $\left[0, \frac{1}{2}\right]$ and $\theta^{v}=\frac{1}{2}$ and another one with $\theta \in\left[\frac{1}{2}, 1\right]$ and $\theta^{v}=\frac{1}{2}$. Let $\underline{q}^{v}$ (resp., $\bar{q}^{v}$ ) be the tempting choice of the former (resp., the later) case. Applying our previous results, we know that the tempting choice in each case is the one located closest to $\theta^{v}$ and the commitment choice satisfies $q(\theta)=\theta$ for all $\theta<\underline{q}^{v}$ and $\theta>\bar{q}^{v}$. Therefore, in this case we have two tempting choices satisfying $V\left(\underline{x}^{v}\right)=V\left(\bar{x}^{v}\right)=0$. Although consumers get the same utility from consuming both tempting choices, we assume that consumer types $\theta \in\left[0, \frac{1}{2}\right]$ consume $\underline{x}^{v}$ while consumer types $\theta \in\left[\frac{1}{2}, 1\right]$ consume $\bar{x}^{v}$. Since $\underline{q}^{v}$ satisfies, $W(\bar{M}, 0)=V\left(\underline{x}^{v}\right)=0$ in equilibrium, $t\left(\underline{q}^{v}, \frac{1}{2}\right)=\pi t\left(\underline{q}^{v}, 0\right)$. Similarly, since $\bar{q}^{v}$ satisfies $W(\bar{M}, 1)=V\left(\bar{x}^{v}\right)=0$ in equilibrium, $t\left(\bar{q}^{v}, \frac{1}{2}\right)=\pi t\left(\bar{q}^{v}, 1\right)$. Graphically
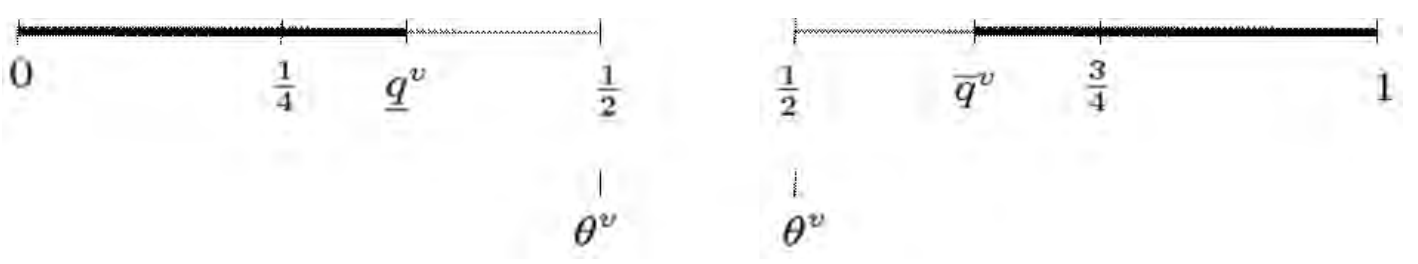

Fig. 4. Optimal Menu when $\theta^{v}=\frac{1}{2}$. 
Since the tempting choices locations are farther away from $\theta^{v}$ as the temptation probability increases, if $\theta^{v} \neq \frac{1}{2}$ it is possible that for a sufficiently large $\pi$, we get a corner solution in $\underline{q}^{v}=0$ or $\bar{q}^{v}=1$, which implies that $t\left(\underline{q}^{v}, \theta^{v}\right) \leq t\left(\bar{q}^{v}, \theta^{v}\right)$ or $t\left(\underline{q}^{v}, \theta^{v}\right) \geq t\left(\bar{q}^{v}, \theta^{v}\right)$ respectively. In the following lemma we summarize this idea

Lemma 6 Let $0 \leq \theta^{v} \leq 1$, in equilibrium

(i) if $\theta^{v}<\frac{1}{2}$

$$
\begin{array}{ll}
t\left(\bar{q}^{v}, \theta^{v}\right)=\pi t\left(\bar{q}^{v}, 1\right)=t\left(\underline{q}^{v}, \theta^{v}\right) & \text { for all } \underline{q}^{v}>0, \\
t\left(\bar{q}^{v}, \theta^{v}\right)=\pi t\left(\bar{q}^{v}, 1\right)>t\left(\underline{q}^{v}, \theta^{v}\right) & \text { for all } \underline{q}^{v}=0,
\end{array}
$$

(ii) if $\theta^{v}>\frac{1}{2}$

$$
\begin{array}{ll}
t\left(\underline{q}^{v}, \theta^{v}\right)=\pi t\left(\underline{q}^{v}, 0\right)=t\left(\bar{q}^{v}, \theta^{v}\right) & \text { for all } \bar{q}^{v}<1, \\
t\left(\underline{q}^{v}, \theta^{v}\right)=\pi t\left(\underline{q}^{v}, 0\right)>t\left(\bar{q}^{v}, \theta^{v}\right) & \text { for all } \bar{q}^{v}=1 .
\end{array}
$$

If $\theta^{v}<\frac{1}{2}$, consumer $\theta=1$ has the lowest incentives to enter the store since his commitment ideal product is the farthest away from his temptation ideal product, thus in equilibrium we have $W(\bar{M}, 1)=0$ to ensure consumers' participation. Moreover, we know that the tempting choices have to satisfy $V\left(\underline{x}^{v}\right)=V\left(\bar{x}^{v}\right)=0$. Therefore, if $\underline{q}^{v}>0$, in equilibrium we have two tempting choices located equidistant from $\theta^{v}$; but, if $\pi$ is so high that it makes $\underline{q}^{v}=0$, we have that $t\left(\underline{q}^{v}, \theta^{v}\right) \leq t\left(\bar{q}^{v}, \theta^{v}\right)$. Therefore, since the monopolist has to make both tempting choices equally desirable for consumers when tempted, he has to charge a lower price for $\underline{q}^{v}$. In particular we have that $\bar{p}=s-t\left(\bar{q}^{v}, \theta^{v}\right) \leq s-t\left(0, \theta^{v}\right)=\underline{p}$. Since $\theta^{v}>\frac{1}{2}$ is the symmetric case, the intuition would be the same.

\section{Uncertainty about the consumers' temptation ideal product, $\theta^{v} \in\left[0, \bar{\theta}^{v}\right]$}

A natural generalization of our model is to consider the case where $\theta^{v}$ is not a single point but rather it takes different values. In particular we consider that $\theta^{v}$ is 
uniformly distributed in the interval $\left[0, \bar{\theta}^{v}\right]$, where $\bar{\theta}^{v} \in(0,1]$. The interpretation is that both, consumers and the monopolist, are unsure about the future temptation ideal product. Let $\underline{q}$ be the product located closest to 0 on the Hotelling line. The following lemma summarizes the equilibrium.

Lemma 7 Let $\theta^{v}$ be uniformly distributed in $\left[0, \bar{\theta}^{v}\right]$, the optimal menu is $\bar{M}=\left\{\theta, p^{*}\right\}$ for all $\theta \in[\underline{q}, 1]$, where $\underline{q}$ satisfies

$$
\begin{gathered}
(1-\pi) t(\underline{q}, 0)+\frac{\pi}{\bar{\theta}^{v}}\left(\underline{q} t(\underline{q}, 0)+\int_{\underline{q}}^{\bar{\theta}^{v}} t(q, 0) d q\right)= \\
\frac{\pi}{\bar{\theta}^{v}}\left[\underline{q} t(\underline{q}, 1)+\int_{\underline{q}}^{\bar{\theta}^{v}} t(q, 1) d q\right] \quad \text { for all } \underline{q}<\bar{\theta}^{v}, \\
t(\underline{q}, 0)=\pi t(\underline{q}, 1) \quad \text { for all } \underline{q}>\bar{\theta}^{v} .
\end{gathered}
$$

and

$$
p^{*}=\left\{\begin{array}{c}
s-p^{*}-(1-\pi) t(\underline{q}, 0)-\frac{\pi}{\bar{\theta}^{v}}\left(\underline{q} t(\underline{q}, 0)+\int_{\underline{q}}^{\bar{\theta}^{v}} t(q, 0) d q\right) \quad \text { for all } \underline{q}<\bar{\theta}^{v} \\
s-t(\underline{q}, 0) \text { for all } \underline{q}>\bar{\theta}^{v} .
\end{array}\right.
$$

Note that if $\underline{q}>\bar{\theta}^{v}$ the result is the same as in the original model, because tempted consumers always choose $\underline{q}$ (i.e., $q^{v}=\underline{q}$ ). Thus, from equation $(6), t(\underline{q}, 0)=\pi t(\underline{q}, 1)$. However, if $\underline{q}<\bar{\theta}^{v}$, every product in the interval $\left[\underline{q}, \bar{\theta}^{v}\right]$ could be chosen under temptation (i.e., $\left.q^{v} \in\left[\underline{q}, \bar{\theta}^{v}\right]\right)$. Therefore, the consumers' ex-ante surplus is

$W(\bar{M} ; \theta)=\left\{\begin{array}{c}s-p-(1-\pi) t(\underline{q}, \theta)-\frac{\pi}{\bar{\theta}^{v}}\left(\underline{q} t(\underline{q}, \theta)+\int_{\underline{q}}^{\bar{\theta}^{v}} t(q, \theta) d q\right) \quad \text { for all } \theta<\underline{q} \\ s-p-\frac{\pi}{\bar{\theta}^{v}}\left(\underline{q} t(\underline{q}, \theta)+\int_{\underline{q}}^{\bar{\theta}^{v}} t(q, \theta) d q\right) \quad \text { for all } \theta>\underline{q}\end{array}\right.$

Note that, knowing $W(\bar{M} ; \theta)$, we only need to require $W(\bar{M} ; 0)=W(\bar{M} ; 1)=0$ to obtain Lemma 7. 

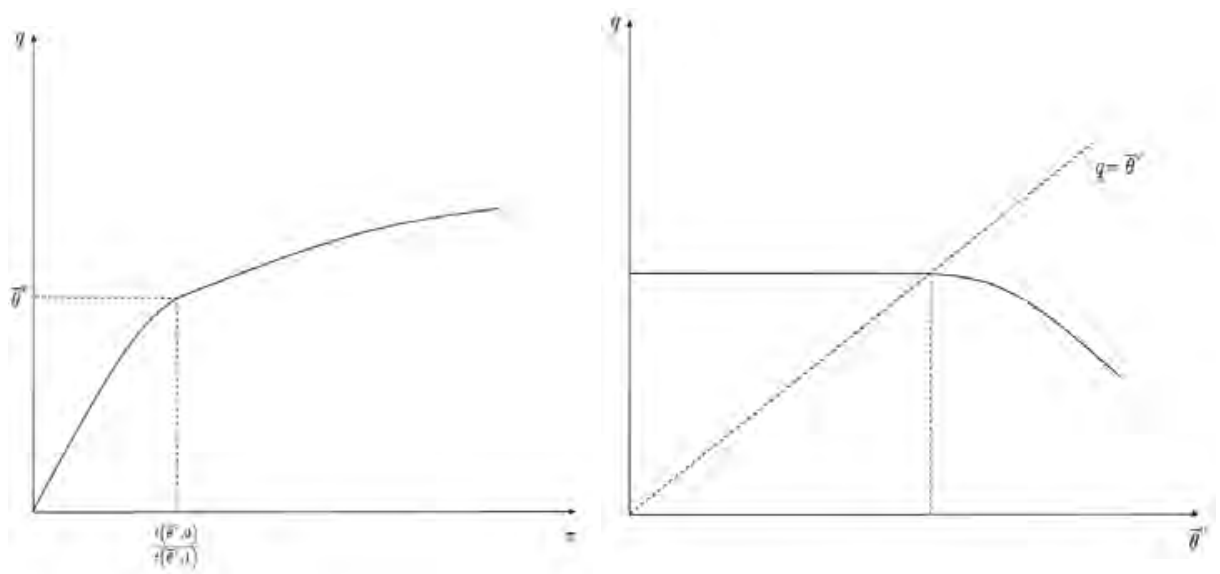

Fig. 5. Equilibrium location $\underline{q}$.

As in the standard case, $q$ is an increasing function of $\pi$. However, note that $q^{v}=\underline{q}\left(\bar{\theta}^{v}=0\right) \geq \underline{q}\left(\bar{\theta}^{v}>0\right)$. The idea behind this is that, when $\underline{q}<\bar{\theta}^{v}$, we do not just have one possible tempting choice but a continuum of possible tempting choices in the interval $\left[\underline{q}, \bar{\theta}^{v}\right]$. Therefore, there are possible tempting choices located closer to $\theta=1$, which gives the consumer a greater ex-ante surplus. This implies that the monopolist can design $\underline{q}$ closer to 0 , which leads to higher product prices. Moreover, the greater $\bar{\theta}^{v}$ is the farther the location of the possible tempting choices will be. The monopolist can farther increase the product prices by lowering $\underline{q}$.

\section{Conclusion}

We have considered a model in which consumers face problems of temptation and selfcontrol, where temptation is modelled as a change of the consumers' ideal product in the Hotelling segment. We have studied the optimal menu designed by a monopolist. 
In our basic model, consumers are perfectly aware of their dynamic inconsistency and all consumers have the same temptation preferences. In this case, the optimal menu is different from the one in a standard horizontal differentiation model in the following sense. In equilibrium, the monopolist truncates the set of products offered, not offering the products closest to the consumers' temptation ideal product. As a result the number of products offered decreases with the temptation probability. As an extension, we have studied the case in which consumers are only partially aware of their dynamic inconsistency. In particular, following Eliaz and Spiegler (2006), we have studied the case in which the monopolist knows that consumers will be tempted but consumers, instead, believe incorrectly that they will be tempted with probability $\pi$. We have shown that, if $\pi$ is sufficiently low, the monopolist offers as many products as consumer types there are, but if $\pi$ is sufficiently high, the firm does not offer the products closest to the consumers' ideal products. Finally, we have studied a more general specification of the temptation preferences and have shown that our main results remain true. 


\section{References}

Bernheim, B. D. and Rangel, A. (2004), "Addiction and Cue-Triggered Decision Processes", National Bureau of Economic Research, Inc.

Brocas, I. and Carrillo, J.D. (2005), "A Theory of Haste", Journal of Economic Behavior \& Organization, 56, 1-23.

Chatterjee, K. and Krishna, R. V. (2005), "Menu Choice, Environmental Cues and Temptation: A "Dual Self" Approach to Self-control", mimeo.

Dekel E., Lipman B.L., Rustichini A. (2005), "Temptation-Driven Preferences", mimeo.

DellaVigna, S. and Malmendier U. (2004), "Contract Design and Self-Control: Theory and Evidence", Quarterly Journal of Economics, 119, 353-402.

Eliaz, K. and Spiegler, R. (2006), "Contracting with Diversely Naive Agents", Review of Economic Studies, 73, 689-714.

Esteban, S., Miyagawa, E. and Shum, M. (2006), "Nonlinear Pricing with SelfControl Preferences", Journal of Economic Theory, 135, 306-338.

Esteban, S. and Miyagawa, E. (2006a), "Optimal Menu of Menus with Self-control Preferences", mimeo.

Esteban, S. and Miyagawa, E. (2006b), "Temptation, Self-control, and Competitive Nonlinear Pricing", Economic Letters, 90, 348-355.

Fudenberg, D. and Levine, D.K. (2006), "A Dual-Self Model of Impulsive Control", American Economic Review, 96, 1449-1476.

Gul, F. and Pesendorfer, W. (2001), "Temptation and Self-control", Econometrica, 69, 1403-1436.

Incekara, E. (2006), "Credit Card Competition and Naive Hyperbolic Consumers", mimeo.

Kreps, D. M. (1979), "A Representation Theorem for "Preference for Flexibility", Econometrica, 47, 565-577. 
Laibson, D. (1997), "Golden Eggs and Hyperbolic Discounting", Quarterly Journal of Economics, 112, 443-477.

Laibson, D. (2001), "A Cue-Theory of Consumption", Quarterly Journal of Economics, 116, 81-120.

O'Donoghue, T. and Rabin, M. (1999a), "Doing It Now or Later", American Economic Review, 89, 103-124.

O’Donoghue, T. and Rabin, M. (1999b), "Incentives for Procastinators", Quarterly Journal of Economics, 114, 769-816.

Sarafidis, Y. (2005), "Inter-temporal Price Discrimination with Time-inconsistent Consumers", mimeo.

Strotz, R. H. (1955), "Myopia and Inconsistency in Dynamic Utility Maximization", The Review of Economic Studies, 23, 165-180. 


\section{APPENDIX \\ Proofs of Propositions and Lemmas}

\section{Proof of Lemma 2}

We show that a gap with no products in $(\underline{\theta}, \bar{\theta}) \subseteq\left[\theta_{k}, 1\right]$, can be improved upon by designing a product with $\widehat{q} \in(\underline{\theta}, \bar{\theta})$. Let $\underline{p}$ be the price of the product $\underline{\theta}$ and $\bar{p}$ be the price of product $\bar{\theta}$. Assume with no loss of generality that consumer $\widehat{\theta} \in(\underline{\theta}, \bar{\theta})$ buys

product $\bar{\theta}$. Then product $\widehat{x}=(\widehat{\theta}, \widehat{p})$ where $\widehat{p}=\max \{\underline{p}+t(\widehat{\theta}, \underline{\theta}) ; \bar{p}+t(\widehat{\theta}, \bar{\theta})\}$, is feasible and yields more profits. Thus, repeating this argument, we get that, in equilibrium, $q(\theta)=\theta$ for all $\theta \in(\underline{\theta}, \bar{\theta})$.

Since $q(\theta)=\theta$ for all $\theta \geq \theta_{k}$ by $U-I C$ constraints we have that $p(\theta)=p^{*}$ for all $\theta \geq \theta_{k}$.

Finally, by $V-I C$ constraint, $p^{*}-p^{v} \geq t\left(q^{v}, \theta^{v}\right)-t\left(\theta_{k}, \theta^{v}\right) \geq 0$, where the last inequality follows from the fact that $q^{v} \leq \theta_{k}$. Q.E.D.

\section{Proof of Lemma 3}

We show that, if $q^{v}<\theta_{k}$, the monopolist finds it optimal to decrease $\theta_{k}$. By definition of $\theta_{k}, U\left(x\left(\theta_{k}\right), \theta_{k}\right)=U\left(x^{v}, \theta_{k}\right)$. Thus, using Lemma $2, p^{v}=p^{*}-t\left(q^{v}, \theta_{k}\right)$. Therefore, the monopolist's profits are

$\Pi=(1-\pi)\left(\theta_{k}\left(p^{*}-t\left(q^{v}, \theta_{k}\right)\right)+\left(1-\theta_{k}\right) p^{*}\right)+\pi\left(p^{*}-t\left(q^{v}, \theta_{k}\right)\right)=p^{*}-t\left(q^{v}, \theta_{k}\right)\left(\pi+(1-\pi) \theta_{k}\right)$, where $\frac{d \Pi}{d \theta_{k}}<0$ for all $q^{v}<\theta_{k}$. Thus, in equilibrium, $q^{v}=\theta_{k}$, which implies $p^{v}=p^{*}$ by incentive compatibility. Q.E.D.

\section{Proof of Proposition 1}

Immediate from Lemmas 1-3. Q.E.D.

\section{Proof of Lemma 4}


Suppose by contradiction that the monopolist designs commitment products in the interval $[\underline{\theta}, 1]$, where $\underline{\theta} \in[0,1)$. Then, for any commitment product, the price is $\underline{p}=s-t(\underline{\theta}, 0)$, which is unique by the incentive compatibility constraints. Therefore, the ex-ante surplus of consumers with $\theta \geq \theta_{k}$, is given by

$$
W(\bar{M}, \theta)=\left\{\begin{array}{c}
(1-\pi)(s-\underline{p}-t(\theta, \underline{\theta}))+\pi\left(t\left(q^{v}, \theta^{v}\right)-t\left(q^{v}, \theta\right)\right) \quad \text { for all } \theta<\underline{\theta}, \\
(1-\pi)(s-\underline{p})+\pi\left(t\left(q^{v}, \theta^{v}\right)-t\left(q^{v}, \theta\right)\right) \quad \text { for all } \theta \geq \underline{\theta} .
\end{array}\right.
$$

Since $\frac{d W(\bar{M}, \theta)}{d \theta}<0$, for all $\theta>q^{v}$, the "worst consumer type" from the point of view of the monopolist is $\theta=1$. Note that by setting $\underline{\theta}=1$ the monopolist maximizes $W(\bar{M}, 1)$ and thus allows for the lowest $q^{v}$ which yields the highest price for the tempting product and hence the highest profits.

Finally, note that since $\underline{\theta}=1, p=s-t(1,0)$, moreover since $V\left(x^{v}\right)=0, p^{v}=$ $s-t\left(q^{v}, 0\right)$. Therefore from the definition of $\theta_{k}, U\left(x\left(\theta_{k}\right), \theta_{k}\right)=U\left(x^{v}, \theta_{k}\right)$ we get that $\theta_{k}=0$. Q.E.D.

\section{Proof of Lemma 5}

Since the monopolist wants to charge the highest possible $p^{v}$, we need to check that $p^{v}=s$ (i.e., locating $q^{v}=\theta^{v}=0$ ) is feasible. In our case, it is sufficient to check that $W(\bar{M}, \theta) \geq 0$ for all $\theta \in[0,1]$

$$
W(\bar{M}, \theta)=(1-\pi)(s-p-t(q, \theta))+\pi\left(s-p^{v}-t\left(q^{v}, \theta\right)\right)
$$

Thus, applying Lemma 4 and letting $p^{v}=s$

$$
\begin{aligned}
W(\bar{M}, \theta) & =(1-\pi)(s-(s-t(1,0))-t(1, \theta))+\pi(t(0,0)-t(0, \theta)) \\
& =(1-\pi)(t(1,0)-t(1, \theta))-\pi t(0, \theta) .
\end{aligned}
$$

Note that

$$
\frac{d W(\bar{M}, \theta)}{d \theta}=0 \text { if and only if }\left|\frac{t^{\prime}(1, \theta)}{t^{\prime}(0, \theta)}\right|=\frac{\pi}{1-\pi}
$$

Due to the properties of the transportation cost function, this implies that for all $\pi \in[0,1], \exists \theta^{*} \in[0,1]$ such that for all $\theta<\theta^{*}\left(\theta>\theta^{*}\right), \frac{d W(\bar{M}, \theta)}{d \theta}>0(<0)$. Therefore, 
since $W(M, 0)=0$, a necessary and sufficient condition for $W(\bar{M}, \theta) \geq 0$ for all $\theta \in[0,1]$ is that $W(\bar{M}, 1) \geq 0$. Since

$$
\begin{aligned}
W(\bar{M}, 1) & =(1-\pi) t(1,0)-\pi t(0,1) \\
& =(1-\pi) t(1,0)-\pi t(1,0)
\end{aligned}
$$

then $W(\bar{M}, 1) \geq 0$ iff $\pi \leq \frac{1}{2}$. This implies that, when $\pi>\frac{1}{2}$, the monopolist has to locate the tempting choice beyond $\theta^{v}$, i.e., $q^{v}>0$, which implies charging a $p^{v}<s$ to attract consumer $\theta=1$ into the store. Since $W(\bar{M}, 1)=0$, then

$$
W(\bar{M}, 1)=(1-\pi) t(1,0)-\pi\left(t\left(q^{v}, 0\right)-t\left(q^{v}, 1\right)\right)=0
$$

Thus

$$
t\left(q^{v}, 1\right)-t\left(q^{v}, 0\right)=\frac{1-\pi}{\pi} t(1,0)
$$

Q.E.D.

\section{Proof of Lemma 6}

It suffices to show $(i)$ since $(i i)$ is symmetric. If $\underline{q}^{v}>0$, the result is trivial. If $\underline{q}^{v}=0$, note that $t\left(\bar{q}^{v}, \theta^{v}\right) \geq t\left(0, \theta^{v}\right)$. If we consider the interval $\left[\theta^{v}, 1\right]$, we can apply the results of the standard case. Thus, in equilibrium, $W(\bar{M}, 1)=V\left(\bar{x}^{v}\right)=0$. Therefore, $t\left(\bar{q}^{v}, \theta^{v}\right)=\pi t\left(\bar{q}^{v}, 1\right)$. Q.E.D.

\section{Proof of Lemma 7}

Note that, in this case, the results of Section 4 (Lemma 2 and Lemma 3) are still valid if we substitute $q^{v}$ for $\underline{q}$. Thus, the optimal menu is $M=\left(\theta, p^{*}\right)$ for all $\theta \in[\underline{q}, 1]$. Moreover, as in Section 4, we have $W(\bar{M}, 0)=W(\bar{M}, 1)=0$ in equilibrium. If $\underline{q}>\bar{\theta}^{v}$, there is just one tempting choice, i.e., $q^{v}=\underline{q}$. Therefore, using the previous condition

$$
s-p^{*}-t(\underline{q}, 0)=(1-\pi)\left(s-p^{*}\right)+\pi\left(s-p^{*}-t(\underline{q}, 1)\right)=0,
$$


so that

$$
t(\underline{q}, 0)=\pi t(\underline{q}, 1) .
$$

However, if $\underline{q}<\bar{\theta}^{v}$, every product in $\left[\underline{q}, \bar{\theta}^{v}\right]$ can be chosen under temptation depending on the realization of $\theta^{v}$. For all $\theta^{v} \in[0, q]$, it follows that $q^{v}=\underline{q}$, whereas for all $\theta^{v} \in\left(\underline{q}, \bar{\theta}^{v}\right], q^{v}=\theta^{v}$. Therefore, since $W(\bar{M}, 0)=W(\bar{M}, 1)=0$ in equilibrium

$$
\begin{gathered}
s-p^{*}-(1-\pi) t(\underline{q}, 0)-\frac{\pi}{\bar{\theta}}\left(\underline{q} t(\underline{q}, 0)+\int_{\underline{q}}^{\bar{\theta}^{v}} t(q, 0) d q\right)= \\
(1-\pi)\left(s-p^{*}\right)+\pi\left(s-p^{*}-\frac{1}{\theta^{v}}\left[\int_{0}^{\underline{q}} t(\underline{q}, 1) d q+\int_{\underline{\underline{\theta}}}^{v} t(q, 1) d q\right]\right)=0 .
\end{gathered}
$$

Thus,

$$
(1-\pi) t(\underline{q}, 0)+\frac{\pi}{\bar{\theta}^{v}}\left(\underline{q} t(\underline{q}, 0)+\int_{\underline{q}}^{\bar{\theta}^{v}} t(q, 0) d q\right)=\frac{\pi}{\bar{\theta}^{v}}\left[\underline{q} t(\underline{q}, 1)+\int_{\underline{q}}^{\bar{\theta}^{v}} t(q, 1) d q\right] .
$$

Finally, since $W(\bar{M}, 0)=0$, then

$$
\begin{aligned}
& p^{*}=\left\{\begin{array}{c}
s-p^{*}-(1-\pi) t(\underline{q}, 0)-\frac{\pi}{\bar{\theta}^{v}}\left(\underline{q} t(\underline{q}, 0)+\int_{\underline{q}}^{\bar{\theta}^{v}} t(q, 0) d q\right) \\
s-t(\underline{q}, 0) \text { for all } \underline{q}>\bar{\theta}^{v} .
\end{array}\right. \\
& \text { Q.E.D. }
\end{aligned}
$$

